\title{
Nitrogen dioxide detection using an optoelectronic sensor
}

\author{
Z. Bielecki ${ }^{1}$, W. Kołosowski ${ }^{1}$, G. Różański ${ }^{1}$, E. Sędek ${ }^{2}$ \\ \& J. Wojtas ${ }^{1}$ \\ ${ }^{I}$ Military University of Technology, Warsaw, Poland \\ ${ }^{2}$ Telecommunications Research Institute, Warsaw, Poland
}

\begin{abstract}
Sensitive laser absorption spectroscopy requires a long effective pathlength of the laser beam in the analyzed media. Traditionally, this requirement is satisfied using an optical multipass cell. We present the opportunities of detection of nitrogen dioxide using cavity enhanced spectroscopies. These techniques can be applied for construction of a fully optoelectronic $\mathrm{NO}_{2}$ sensor with a detection limit better than $1 \mathrm{ppb}$. The setup usually includes the resonance optical cavity, which is equipped with spherical and high reflectance mirrors, the pulsed or cw laser and a photomultiplier which is connected with the digital oscilloscope or with others digitizers. The optical signal leaving a cavity can be used to determine an absorption coefficient of the intracavity medium.
\end{abstract}

Keywords: optoelectronic sensor, absorption spectroscopy.

\section{Introduction}

Nitrogen dioxide is one of the most important factors in atmosphere quality. At present, $\mathrm{NO}_{2}$ is commonly detected using the methods based on chemiluminescence. Their sensitivity reaches single ppb.

Laser absorption spectroscopy is an effective tool in studies of gaseous species. The Beer-Lambert law for week absorption indicates that the minimum detectable concentration of absorbers is inversely proportional to the effective sample-path length, and directly proportional to the minimum intensity fluctuation detected by a photodetector. In order to increase the absorption sensitivity, the optical path through the absorber should be maximised and the relative-intensity variations should be minimised. 

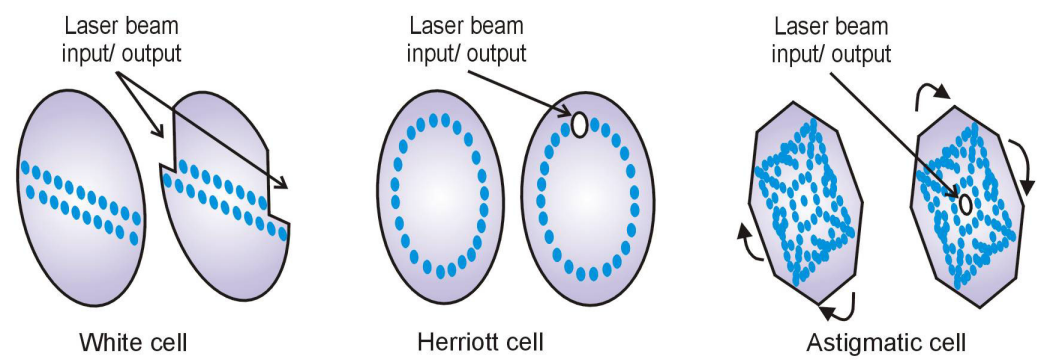

Figure 1: $\quad$ Example of commonly used optical multipass cell.

Long optical path lengths for absorption can be achieved by the use of White or Herriott cells (Fig. 1), a passive high-finesse cavity or laser intracavity technique [1-3].

An effective absorption path length up to several kilometres has been obtained using laser intracavity absorption spectroscopy. Use of a high-finesse cavity permits hundreds, or even thousands, of traverses through the absorber, and thus reaches long effective path lengths and provides high detection sensitivity.

One of the methods of laser intracavity absorption spectroscopy is cavity ring-down spectroscopy (CRDS). The CRDS based on the measurement of the time for light to decay within the cavity, has been developed for use with a pulsed laser (P-CRDS) or with a continuous-wave laser (CW-CRDS) with acousto-optic modulator or mechanical beam chopper (Fig. 2) [4, 5].

There are others methods using a high-finesse optical cavity consisting of two highly reflective mirrors $(\mathrm{R}>0.9999)$ such as cavity enhanced absorption spectroscopy (CEAS) with off-axis laser and cavity adjustment, integrated cavity output spectroscopy (ICOS) with piezoelement or ring-down spectral photography (RSP) [5-8].

\section{Experimental design}

The laser intracavity absorption spectroscopy techniques share a common instrument layout. A basic schematic layout of the instrument design is shown in Fig. 2. In all cases, a laser light is coupled into high-finesse optical cavity, which is equipped with gas valves. The valves are connected with the gas mixing system, which allows for precise gas mixing and establishing the ordered $\mathrm{NO}_{2}$ concentration.

The laser radiation can be directed to the cavity using two mirrors. Due to pulsed character of the radiation used, one can avoid various problems with the light modulation and the laser - cavity mode coupling.

A photodetector, often PMT, monitors the power escaping from the cavity, which is proportional to the intensity inside a cavity. PMT can be equipped with the interference filter, the bandpass of which was well matched to the laser line. 


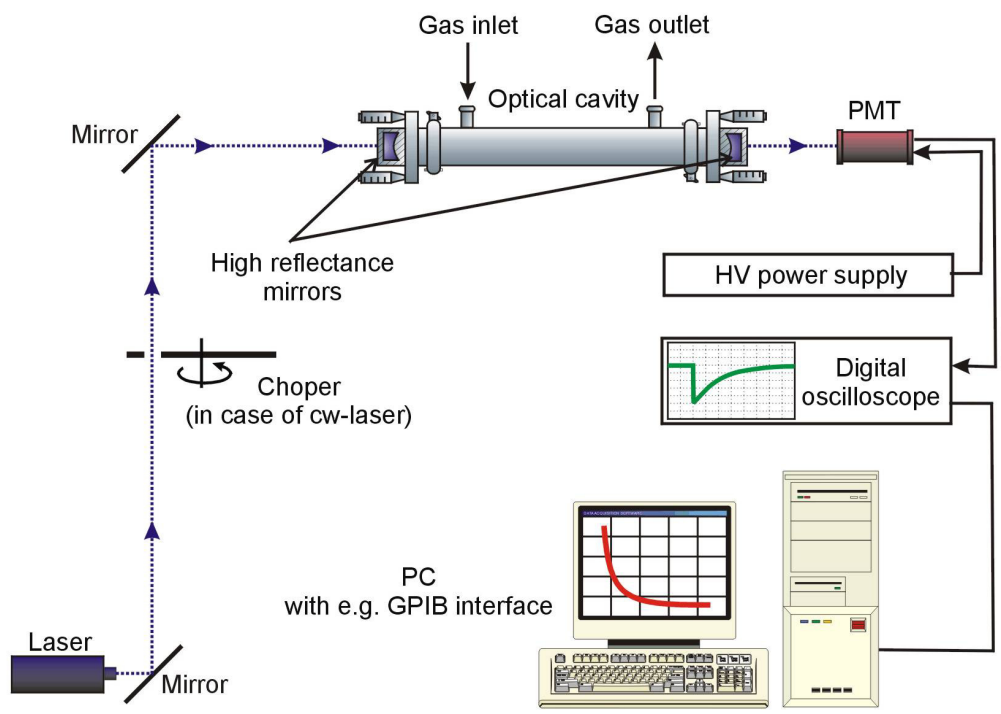

Figure 2: $\quad$ Example of CRDS setup.

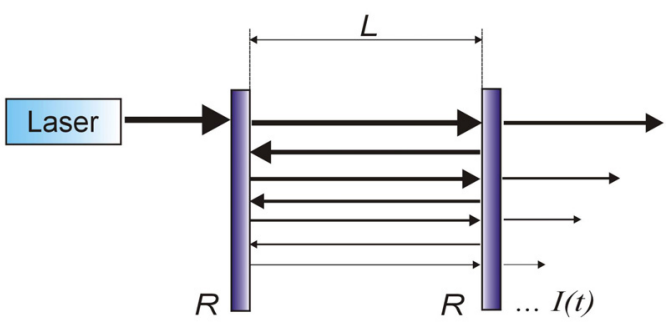

Figure 3: $\quad$ Idea of CRDS.

Next, the signal is recorded with a fast digital oscilloscope and after digitization usually a computer is used to store and analyze the measurement data.

\section{Analyses}

In CRDS technique, the gas sample is placed inside a high-finesse optical cavity. It consists of two highly reflective mirrors. A short laser pulse is coupled into the cavity. The light is reflected back and forth inside the cavity and, every time that the light is reflected, a small fraction of this light leaks out of the cavity and is registered by a photodetector (Fig. 3). 
The presence of absorbing species in the cavity gives an additional loss channel for the light inside the cavity. If the absorption follows Beer's law, the light intensity inside the cavity will still decay exponentially, resulting in a decrease in the decay time as

$$
I(t)=I_{0} e^{-\frac{[(1-R)+\alpha L] c}{L} t}=I_{0} e^{-\frac{t}{\tau}},
$$

where $I_{0}$ is the initial intensity, $c$ denotes the light speed, $\alpha$ is the absorption coefficient, and $L$ is the resonator length.

The decay time of radiation is measured once when the cavity is empty (Fig. 4, dashed line B) and next when the cavity is filled with the absorber (Fig. 3, line A).

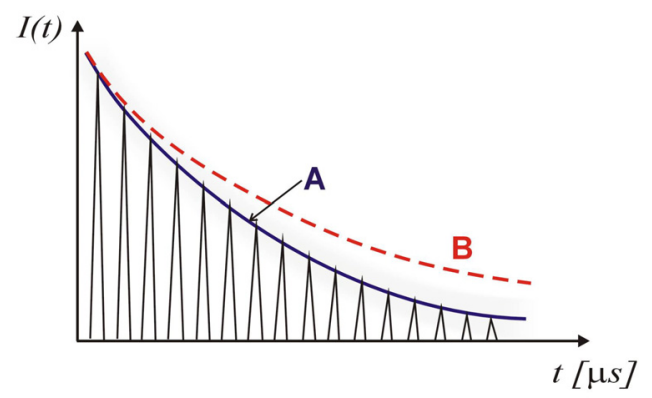

Figure 4: Decay signal from a cavity.

In an empty cavity, this ring-down transient is a single-exponentially decaying function of time with a 1/e CRDS time which is solely determined by the reflectivity of the mirrors and the optical path length between the mirrors.

Speed of the decay intensity $I(t)$ of the pulse of the laser light is dependent on the mirrors reflectivity coefficient $R$, the resonator length $L$, diffraction losses, and extinction, that is absorption and scattering of a light in the absorber filled cavity. Therefore, by measuring the resonator quality, determination of the absorption coefficient is possible. The resonator quality can be determined by measurement of the radiation decay time constant $\tau$

$$
\tau=\frac{L}{c \cdot[(1-R)+\alpha L]} .
$$

By comparison of decay times for these two cases, a value of the absorber density $N$ can be found

$$
N=\frac{\alpha}{\sigma}=\frac{1}{\sigma c}\left(\frac{1}{\tau}-\frac{1}{\tau_{0}}\right),
$$

where $\sigma$ denotes the absorption cross section, while $\tau_{0}$ and $\tau$ are the time constants of the exponential decay of the output signal for empty resonator and for the resonator filled with the absorber, respectively. 
When the high reflectivity mirrors $(\mathrm{R}>0.9999)$ are applied, the absorption coefficients as low as $10^{-9} \mathrm{~m}^{-1}$ can be measured.

There are several advantages to this approach. Since the absorption is determined from the time behaviour of the signal, it is independent of pulse-topulse fluctuation of the laser. Furthermore, the effective absorption path length, which depends on the reflectivity of the cavity mirrors, can be very long (several $\mathrm{km}$ ), while the sample volume can be kept rather small. Another advantage is that the absorption is measured on the absolute scale. The CRDS technique can be applied with success when the molecule's excited state does not fluoresce or can be ionized. In high-pressure samples, such as plasmas and flames, CRDS can be successfully used to extract quantitative absolute concentration data, which is nearly impossible by using other methods. As long as the mirrors with a sufficiently high reflectivity, detectors with a sufficiently sensitivity and fast time response, and tunable light sources are available, there is no intrinsic limitation to the spectral region in which CRDS can be applied. This method can be used from the ultraviolet part of the spectrum to the infrared spectral region.

\section{Nitrogen dioxide concentration measurement}

The nitrogen dioxide absorption cross section $\sigma$ is shown in Fig. 5 [9]. The greatest $\sigma$ values reached around $400 \mathrm{~nm}$. There are several minima and maxima, but they varying about value of $6 \times 10^{-19} \mathrm{~cm}^{2}$.

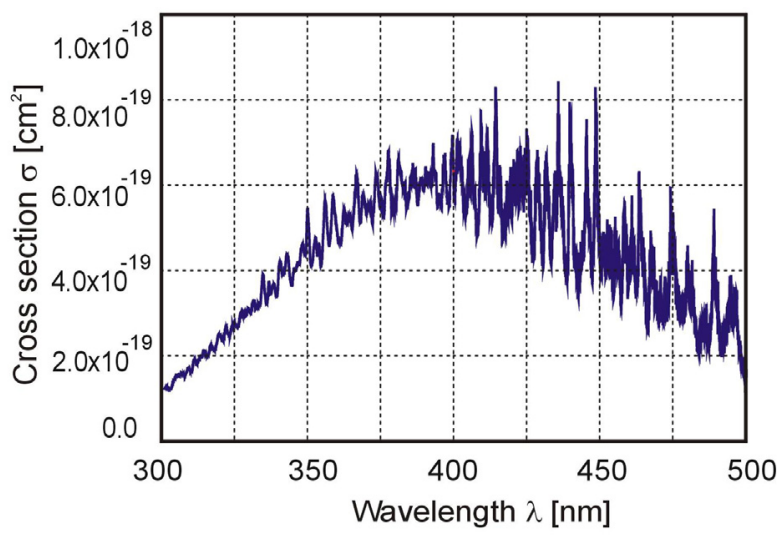

Figure 5: $\quad \mathrm{NO}_{2}$ absorption cross section.

In order to achieve a high value of limit detection of $\mathrm{NO}_{2}$ reflectivity, cavity mirrors and wavelength of a laser beam should be appropriately matched to the greatest values of nitrogen dioxide absorption cross section.

Furthermore, to achieve long decay times and high precision of the decay time determination, the proper adjustment of the cavity is necessary. Moreover, the measurement with a good detection limit also requires appropriate filtration 
of the investigated air. This is necessary in order to avoid the light scattering in the aerosol particles as well as the dust deposition on the mirror surfaces. When the concentration of aerosol particles in gas is similar to that in free atmosphere, the extinction of about $10^{-6} \mathrm{~m}^{-1}$ occurs, consequently at high sensitivity measurements, the losses due to the light scattering can be the same or larger than the absorption losses.

Sensitivity of nitrogen dioxide optoelectronic sensor based on cavity enhanced spectroscopy technique is determined by detectable concentration limit $N_{\text {Limit }}$, which is described by the formula

$$
N_{\text {Limit }}=\frac{X}{c \sigma(\lambda) \tau_{0}},
$$

where $X$ is the relative precision of time determination, and is equal to

$$
X=\frac{\tau_{0}-\tau}{\tau_{0}} .
$$

In Fig. 6, the dependence of sensitivity nitrogen dioxide sensor on cavity length and mirrors reflectivity is shown. The calculations were performed for $5 \%$ of decay time precision determination and absorption cross section equaled to $5.1 \mathrm{~cm}^{2}$. As it is shown in the picture, strong influence on sensor sensitivity has mirrors reflectivity.

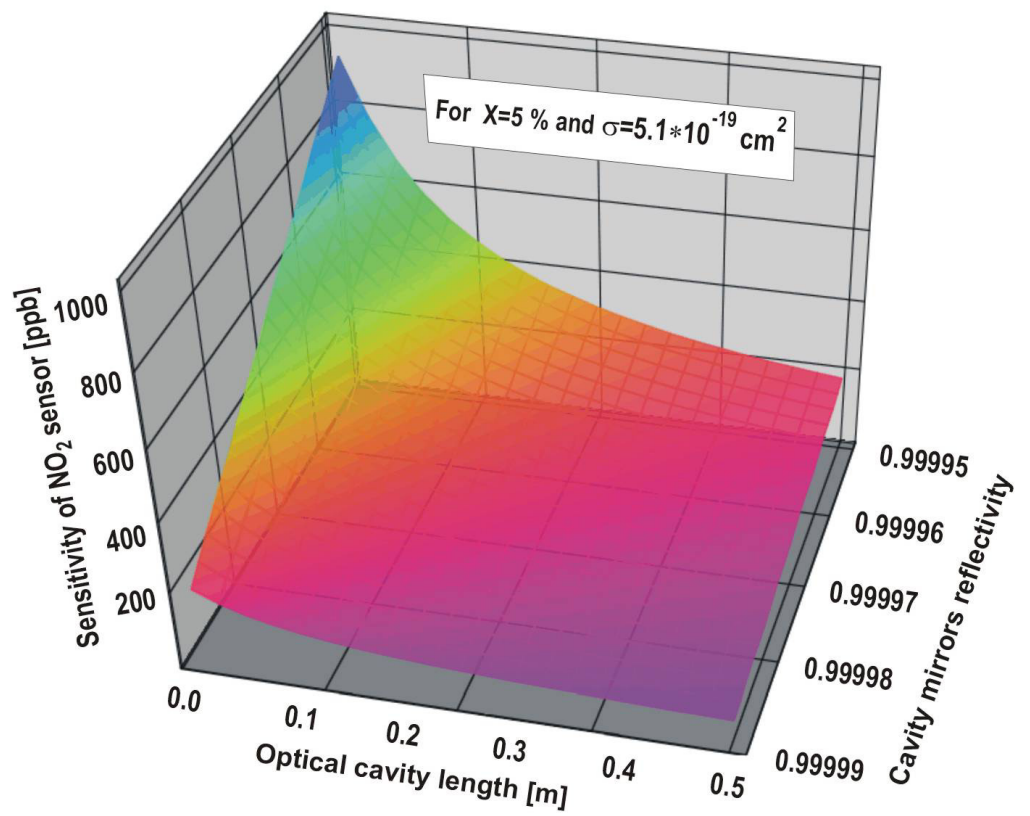

Figure 6: Dependence of $\mathrm{NO}_{2}$ sensor sensitivity on cavity length and mirrors reflectivity. 
Detectable concentration limit is also strongly biased by decay time precision determination. For instance, if $1 \%$ precision is ensured, sensor with mirrors, the reflectivity of which reached a value of $99.995 \%$, provides possibility of measurement of $0.5 \mathrm{ppb}$ of nitrogen dioxide, what is presented in Fig. 7. The dependence of $\mathrm{NO}_{2}$ sensor sensitivity on decay time precision determination and cavity mirrors reflectivity is shown.

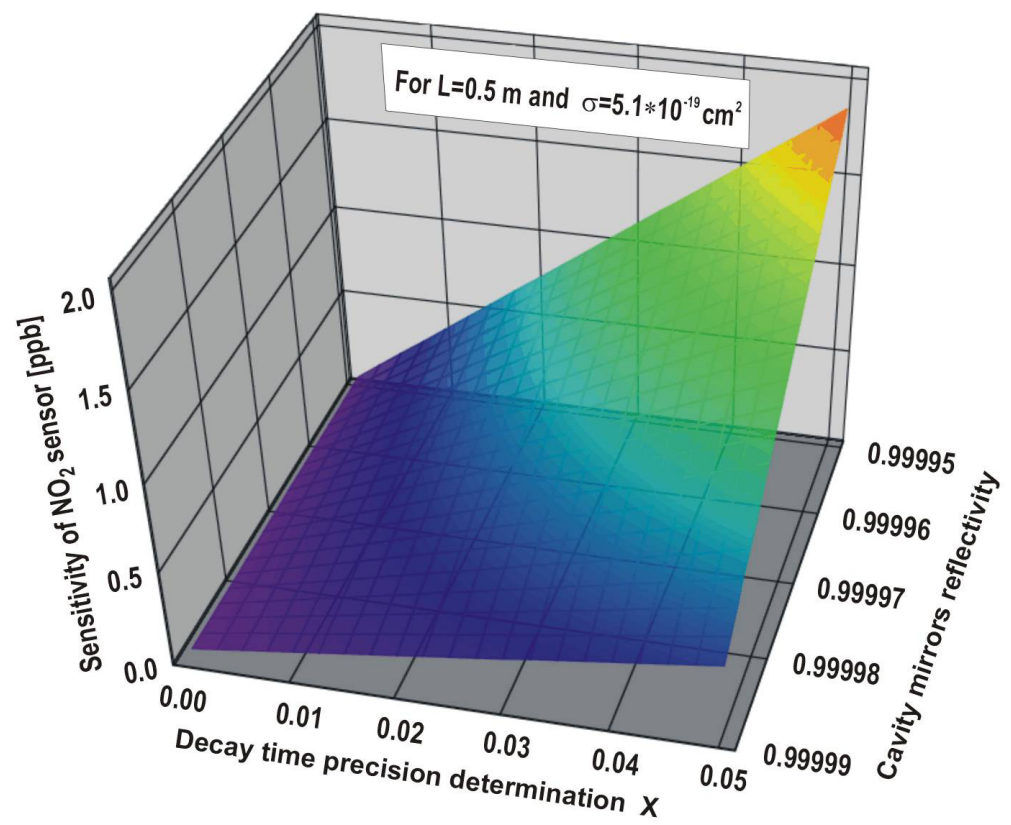

Figure 7: Dependence of $\mathrm{NO}_{2}$ sensor sensitivity on decay time precision determination and cavity mirrors reflectivity.

It is possible to reach highly precise decay time determination using coherence averaging of samples of signal from a photodetector [10]. Experiment shows, that thanks to this precision better than $0.2 \%$ is attainable after the averaged 10000 CRDS pulses (Fig. 8).

\section{Conclusions}

In this paper, we have presented cavity enhanced spectroscopic technique which can be applied for construction of a fully optoelectronic $\mathrm{NO}_{2}$ detection system. A manner of resonator quality determination by measuring the time of the radiation imprisonment is not sensitive for the laser power fluctuation and photodetector sensitivity fluctuation. 


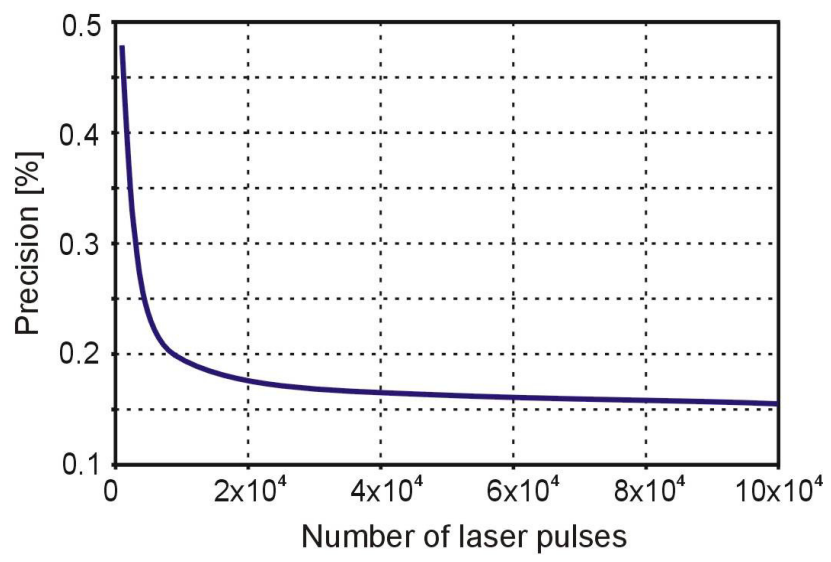

Figure 8: Precision of decay time determination as a function of a pulses number.

The features of the described detection system show, that it is possible to construct a $\mathrm{NO}_{2}$ sensor, the sensitivity of which could be comparable with that of chemical detectors. Such a kind of system has several advantages such as: low price, small size and weight and possibility of detection of other gases.

In the next stage of the experiment we are going to construct a fully optoelectronic system based on cavity enhanced spectroscopic technique for detection of trace concentration nitrogen dioxide.

\section{References}

[1] J.U. White, Very long optical paths in air, J. Opt. Soc. Am. 66, 411, 1976.

[2] D. Herriott, H. Kogelnik, R. Kompfner, Off-axis paths in spherical mirror interferometers, Applied Optics, 3, p. 523, 1964.

[3] J. Doussin, R. Dominique, C. Patrick, Multiple-pass cell for very-longpath infrared spectrometry, Applied Optics, Vol. 38, No. 19, pp. 4145 $4151,1999$.

[4] A. O'Keefe, D.A.G. Deacon, “Cavity ring-down optical spectrometer for absorption measurements using pulsed laser sources”, Rev. Sci. Instrum. 59, No. 12, pp. $2544-2551,1988$.

[5] G.S. Engel, E.J. Moyer, F.N. Ketusch, J.G. Anderson, Innovations in Cavity Enhanced Laser Absorption Spectroscopy: Using in situ Measurements to Probe the Mechanisms Driving Climate Change, Earth Science Technology Conference, Laser Sensor Technologies, June, 2003.

[6] J.J. Scherer, Ringdown spectral photography, Chemical Physics Letters, No. 292, pp. 143-153, 1998.

[7] J.J. Scherer, J.B. Paul, H. Jiao, A. O'Keefe, Broadband ringdown spectral photography, Applied Optics, Vol. 40, No. 36, pp. 6725-6732, 2001. 
[8] R.F. Curl, F.K. Tittel, Tunable infrared laser spectroscopy, Annu. Rep. Prog. Chem., Sect. C, 98, pp. 219-272, 2002.

[9] M.F. Merienne, A Jenouvrier, B. Coquart, The $\mathrm{NO}_{2}$ absorption spectrum. I: absorption cross-sections at ambient temperature in the $300-500 \mathrm{~nm}$ region, J. Atmos. Chem., Vol. 20, No. 3, pp. 281-297, 1995.

[10] J. Wojtas, A. Czyżewski, T. Stacewicz, Z. Bielecki, J. Mikolajczyk, Cavity enhanced spectroscopy for $\mathrm{NO}_{2}$ detection, Proc. SPIE, Vol. 5954, pp. 174-178, 2005. 